\title{
Relación del perfil profesional con la contratación de profesionales en administración de empresas hoteleras de la Universidad Internacional del Ecuador - extensión Guayaquil en el año 2015
}

\section{Relación del perfil profesional con la contratación de profesionales en administración de empresas hoteleras de la Universidad Internacional del Ecuador - extensión Guayaquil en el año 2015}

\author{
Ana Luisa López Brunett \\ Edgar Larco Camacho \\ Carlos Ortiz Chimbo \\ Universidad Internacional del Ecuador, Ecuador
}

Autor para correspondencia: anlopezbr@mail.internacional.edu.ec, edlarcoca@uide.edu.ec, caortizch@internacional.edu.ec.

Fecha de recepción: 20 de Octubre - Fecha de aceptación: 20 de diciembre de 2016.

\begin{abstract}
Resumen
Mediante la presente investigación se ha determinado la relación existente entre el perfil profesional con el que se gradúan los estudiantes de la carrera de Administración de Empresas Hoteleras de la Universidad Internacional del Ecuador, extensión Guayaquil, con la contratación de profesionales en el sector turístico. Se levantaron encuestas a los graduados de la carrera y, de esta manera, se pudo evaluar y contrastar el perfil profesional obtenido en el transcurso de su vida estudiantil con los perfiles que requiere y demanda el mercado laboral turístico. Los resultados obtenidos fueron esclarecedores de varias de las causas o factores que influyen de manera positiva o negativa en la relación entre el perfil profesional y la inserción laboral.
\end{abstract}

Palabras claves: Perfil profesional; contratación de profesionales; turismo y hotelería; competencias generales

\begin{abstract}
Through this research we have determined the relationship between the professional profiles with students Administration de Empresas Hoteleras de la University International del Ecuador, extension Guayaquil, hiring professionals in tourism graduate. For this survey rose to graduates of this race and thus is able to evaluate and contrast the professional profile obtained during their student life with profiles that requires and demands the tourism labor market. The results were illuminating more of the causes or factors that influence positively or negatively on the relationship between the professional profile and job placement.
\end{abstract}

Key Words: Professional profile; hiring professionals; tourism and hospitality; general skills 


\section{Introducción}

\section{Análisis del Perfil Profesional en el mercado laboral}

El sector turístico y hotelero es cada vez más competitivo en el mundo entero. A nivel interno, en el Ecuador, el turismo se ha desarrollado proporcionando nuevas plazas de trabajo a un mercado de talento humano que desea ofrecer sus servicios profesionales.

El perfil profesional de un estudiante graduado conlleva varios puntos importantes y necesarios que van a diferenciar a un profesional de otro, como lo son sus conocimientos, habilidades, destrezas y valores, es decir, sus competencias generales adquiridas en el transcurso de su carrera universitaria, las cuales van a ser demostradas en el desenvolvimiento y desarrollo del puesto de trabajo para el cual haya logrado ser contratado.

Al pensar en la relación que existe entre el perfil profesional de un ingeniero en Administración de Empresas Hoteleras con la contratación de profesionales en el mercado turístico, es necesario analizar el perfil por competencias que adquiere el estudiante en sus años de estudio universitario, su desempeño ante diversos contratiempos, sus capacidades, conocimientos, habilidades y demás que son los requisitos solicitados por las empresas al momento de contratar profesionales, y que cada vez se tornan más exigentes.

El Ministerio de Turismo indica que el año 2014 cerró con 119.296 empleados (56.444 mujeres y 62.852 hombres) trabajando en el sector turístico, que forman parte de los establecimientos registrados en el catastro del MINTUR. Con base en ese dato, se estimó que el 2015 cerraría con un total de 133.081 personas trabajando en el área aproximadamente, cifra calculada en base a la tasa de crecimiento anual de los empleos directos en turismo del 11,74\% para los hombres y 11,35\% para las mujeres. Dentro de este grupo se pueden encontrar varios tipos de colaboradores, ya que están los que tienen conocimientos empíricos en la profesión, los que estudiaron administración de empresas en general, mas no turística, y que decidieron ingresar al medio ya sea por apostar a un nuevo negocio o porque el factor necesidad los obligó. Los titulados en alguna rama del turismo que se desempeñan eficientemente en sus trabajos afines y también los que tuvieron ayuda por parte de algún conocido para ingresar a un puesto de trabajo en el área. Esto conduce a evaluar la relación del perfil del estudiante con la contratación de profesionales, y cuyos resultados coadyuven a realizar cambios en el perfil por competencias de la carrera.

Acuña \& Mozombite (2013) plantean la cuestión “¿Qué tan preparados están hoy en día los jóvenes al terminar sus estudios superiores, teniendo en cuenta la competitividad laboral?”. La competencia de talentos para encontrar un puesto de trabajo en el área se intensifica, y cada uno de ellos se va a diferenciar por su grado de preparación recibida en su vida estudiantil.

En cuanto al perfil profesional del egresado, Pavié (2012) afirma que es "el conjunto de competencias, rasgos y capacidades que, certificadas apropiadamente, permiten que alguien sea reconocido por la sociedad como profesional". En cuanto a ello, el perfil profesional que posee un egresado, sea cual fuere su rama de estudio, es aquel que lo va a caracterizar y diferenciar de los demás, ya que posee conocimientos y habilidades propias obtenidas a lo largo de su carrera, 
que le permitirá encontrar un puesto laboral acorde a dicho perfil para el cual se encuentra competente y capacitado.

De manera similar, Ayala, Castillo \& Hernández (2011) señalan que “el perfil del profesional está compuesto por el conjunto de capacidades y competencias que identifican la formación de una persona para asumir en condiciones óptimas las responsabilidades propias del desarrollo de funciones y tareas de una determinada profesión”. Dicho perfil profesional se lo forma conforme se va desarrollando la vida estudiantil del graduado, adquiriendo las competencias necesarias como conocimientos, habilidades, destrezas, valores, entre otros, que diferenciarán a un profesional de otro. Es la marca propia.

Jara (2010) manifiesta que el perfil profesional debe contar con requerimientos básicos o fundamentales, que son las condiciones imprescindibles sin las cuales será imposible un rendimiento normal en el puesto de trabajo, y requerimientos complementarios, que son las características que al ser combinadas con los requisitos básicos, permitirían un rendimiento óptimo en el puesto. Ambos requerimientos son necesarios dentro del perfil del graduado, para que exista una sinergia entre ambos y se complementen, convirtiendo al profesional en alguien destacado.

La definición del perfil posee una especial importancia por cuanto va actuar como un punto de referencia y guía de todo el resto del proceso. Los contenidos que se seleccionen, las prácticas que se incorporen, la secuencia en que todo ello se integra van a estar condicionada por el tipo de perfil profesional al que esté vinculado un determinado plan de estudios. (Zabalza, 2007). Los contenidos que se incluyan en un plan de estudios deben estar encaminados al perfil profesional que se quiera alcanzar, incluyendo las materias a estudiarse, las prácticas en el campo de estudio para obtener experiencia, los trabajos y las investigaciones a realizar por el estudiante, para desarrollar con precisión las competencias necesarias que le serán de mucha utilidad en su vida laboral.

Tradicionalmente la formación impartida en las universidades españolas se ha caracterizado por la transmisión de conocimientos, la formación de profesionales especializados en un determinado ámbito relacionado con un perfil profesional, para el que la titulación obtenida acredita que un titulado puede ejercer, si lo desea, una profesión. (García M. R., 2006).

En otros países como España, se afirma que la formación universitaria que reciben los estudiantes de sus instituciones de tercer nivel, le son suficientes y llenan las expectativas de los mismos al momento de desarrollar su profesión para el cual fueron preparados. Aseguran un perfil óptimo y competente.

Llanos (2008), señala que "el desempeño eficiente de una profesión no es otra cosa que la correcta aplicación de lo adquirido en la formación profesional". Es claro que cuando existe una formación profesional correcta, se ve reflejada en las actividades que realiza un graduado en su puesto de trabajo, desempeñadas con eficiencia y responsabilidad. Las empresas hoy en día requieren profesionales con determinados perfiles; no basta con egresar o tener un título profesional, hay que desarrollar competencias y habilidades que hacen a un profesional realmente competente en el mercado laboral. (Acuña \& Mozombite, 2013). 
Obtener un título profesional no garantiza que el graduado esté en las condiciones necesarias para desempeñarse en el mundo laboral. Como se indica, es necesario que desarrolle sus competencias y habilidades mediante la experiencia obtenida en su carrera universitaria, en sus pasantías pre profesionales y en algún empleo previo que haya obtenido ya que, hoy en día, existe una alta competencia en el mercado laboral y las empresas solicitan personas más preparadas y con mayor grado de experiencia.

Albornoz \& Escudero (2007) afirman que "la descripción de un perfil profesional es importante en el diseño y evaluación del currículo, ya que aporta consistencia y validez. Además, ofrece respuestas del campo ocupacional en relación a las necesidades de empleadores para contar con estos profesionales", lo que indica que dicho perfil es la parte medular que define el grado de profesionalismo con el que cuenta el aspirante a un determinado puesto de trabajo. El empleador va a considerar mucho este punto para poder tomar una decisión frente a la cantidad de postulantes que se presenten al cargo. Ortiz (2013), indica que "se puede evidenciar que el perfil profesional que obtiene un egresado del área de turismo, aporta al buen desempeño y planificación de las instituciones y empresas turísticas". Varias son las universidades que ofrecen títulos de licenciatura en Turismo, cada una de ellas diferencian sus mallas con ciertas materias, dependiendo de la especialidad que se derive de la misma, como Turismo y Hotelería, Turismo y Medio Ambiente, Turismo y Transportes turísticos, entre otras. La institución asegura que el estudiante graduado posee los conocimientos y habilidades necesarias para insertarse en el mercado turístico para el cual se preparó, esperando que apenas obtenga su título pueda ocupar un cargo como mínimo a nivel de mandos medios (jefe). Esto se va a ver influenciado por diversos factores como experiencia mínima requerida, puestos vacantes disponibles y fuerte competencia con otros graduados.

Dentro del perfil del egresado, se debe contar con un óptimo nivel en conocimientos del sistema turístico, de infraestructura hotelera, de rutas y circuitos y dominio de un idioma extra al de su lengua natal, normalmente el idioma inglés. (Carrión, 2013).

Al vincular el sistema turístico ocupacional, educacional y el científico - tecnológico, permite que el profesional en turismo perciba la realidad en la que va a desempeñar sus funciones al insertarse en el mundo laboral, según señala Gómez (2009). El mercado turístico se desarrolla cada vez más, y las innovaciones están a la orden del día, por ello es necesario que el profesional observe todos los aspectos que lo rodean y logre formar una conexión entre los mismos para colaborar positivamente dentro del sector.

Por otra parte, la Universidad Autónoma de Nayarit (2014) señala que "la formación profesional en la dinámica actual, debe impulsar la incorporación de mayores niveles de conocimiento, fomento de trabajo en equipo, capacidad de interacción simbólica, conocimiento amplio del proceso educativo, desarrollo de un pensamiento innovador, así como mentalidades críticas y propositivas". Está claro que el conocimiento y las habilidades que se adquieren a través de un perfil de competencias son muy importantes para el correcto desenvolvimiento del graduado en cualquier rama del Turismo. El saber relacionarse con los demás, proponer nuevas ideas, ser un líder, entre otras características más son las competencias que deben formar parte de un perfil profesional. 


\section{La Contratación de Profesionales partiendo de las Practicas Pre profesionales}

La inserción al mercado laboral de un graduado en Turismo y Hotelería resulta muchas veces complicada, ya que existe un exceso de oferta de mano de obra y muy pocos puestos de trabajo disponibles en el área. Para Souza \& Luce (2007) "la formación e inserción profesional en el ámbito turístico se encuentra llena de problemas que necesitan ser discutidos. Hasta el momento se han realizado pocas investigaciones para conocer la inserción profesional de los egresados de las carreras de turismo". Además los requisitos que piden ciertas empresas para contratar no son alcanzados por dichos graduados, disminuyendo en ellos las ganas de desenvolverse en la industria turística.

La relación existente entre el mercado laboral y la formación académica ha venido deteriorándose en los últimos años, ya que es normal encontrar a un graduado en Turismo trabajando en una empresa de telecomunicaciones o de construcción, razón por la cual es necesario que exista un cambio o un rediseño de los perfiles por competencias académicas de los egresados en Turismo y de sus respectivas mallas curriculares. Dicha relación entre los ámbitos profesional y académico debe ser cambiada para beneficio de los graduados y sus profesiones. (Peñaloza, Medina, Herrera, \& Vargas, 2011).

Es importante mencionar que las prácticas pre profesionales o pasantías que los estudiantes de la carrera realizan como requisito indispensable para su graduación, contribuyen también a la formación profesional de los mismos. En varias ocasiones, los estudiantes luego de haber culminado sus horas de pasantías, continúan desempeñando sus funciones en la empresa en donde las realizan, es decir, son contratados formalmente por la misma, llegando a formar parte de los colaboradores de planta. Los que no contaron con esa suerte, pueden utilizar esa experiencia profesional para obtener empleos similares. Como los autores indican "aquellos que se incorporan al mundo laboral se sentían mejor valorados que sus compañeros tanto por parte de sus superiores como por los compañeros con otras titulaciones". (Crous-Costa \& Vidal-Casellas, 2013).

De igual manera lo afirma Martínez (2005) "la inserción laboral, por tanto, se ha convertido en uno de los principales objetivos a conseguir con la realización de prácticas en empresas", lo que confirma que las pasantías profesionales que realizan los estudiantes en su vida universitaria, coadyuvan a que puedan insertarse de manera más rápida en el mundo laboral. Es muy importante que las universidades tengan en cuenta este punto y firmen convenios con empresas que estén relacionadas con las carreras.

La primera experiencia en el mundo laboral de un estudiante universitario, normalmente se da en su periodo de pasantías profesionales, en donde debe aprender a desarrollar habilidades y conocimientos mediante las actividades que se le han encomendado. Aun así, este tema no resulta fácil para muchos de ellos, inclusive para las empresas, quienes también le dan la importancia que se necesita al querer incorporar pasantes a sus instalaciones. Dicho pasante debe demostrar su responsabilidad y profesionalismo, ya que puede estar desempeñándose en el que podría ser su trabajo fijo en un futuro. El estudiante graduado, al salir al mundo laboral, puede empezar por lo más básico que es la entrega de sus hojas de vida en físico o enviarlas a través de 
correo electrónico. Algunos de ellos no dudan en aceptar puestos bajos u operativos para comenzar su carrera, ya que a lo largo de la misma van obteniendo experiencia, y tienen la posibilidad de ascender de puesto a nivel interno. Luego de ello deciden si se mantienen laborando donde están o si desean trabajar independientemente. (Dieckow \& Kremar, 2014).

No se puede responsabilizar al Sistema Educativo del fracaso de la inserción laboral de los jóvenes egresados, la tasa de crecimiento de la economía es el factor central de la capacidad para crear empleos. (García C. , 2009) dicha autora intenta demostrar otro punto importante dentro de la problemática en la inserción laboral de los graduados en general, que indica que la economía de cada país también influye a que los egresados no puedan encontrar un puesto de trabajo, puesto a que son las empresas privadas las que generan nuevas vacantes, y en vista de que existe cierta desconfianza para invertir en la actualidad, se disminuye el porcentaje de nuevos negocios en el Ecuador y en ciertos territorios.

En otras universidades del mundo ya se han realizado estudios de mercado laboral, que han reflejado información valiosa que ayuda a mejorar los planes de estudio de las carreras que se imparten en las mismas, proporcionando conocimientos, habilidades y destrezas que a su vez contribuyen a mejorar el nivel de inserción laboral de los egresados. (Segundo, 2009).

Pereira (2012) indica que "claramente podemos observar que el panorama laboral es cada vez más exigente, que requiere profesionales con la cualificación del nivel más avanzado, que ha excluido a muchos jóvenes y adultos en el mercado laboral". Esto se encuentra ligado a la modernización, a la globalización y a las innovaciones que se dan constantemente en la sociedad, por ello el mercado laboral exige una mejor preparación en los jóvenes graduados de las instituciones de tercer nivel en la industria del turismo y en las demás. Esta es otra de las razones por las que se suele dificultar la inserción laboral.

La problemática del empleo ha estado sujeta a numerosas intervenciones estatales y la mayoría de los países aspiran a conseguir el pleno empleo, es decir, a que todos quienes quieran trabajar puedan hacerlo. (Zalba, 2004). El autor indica que el Estado también ha intervenido en la obtención de empleos en un país, en varios casos afectando a los estudiantes graduados o beneficiándolos, dependiendo del Gobierno. En Ecuador se está dando facilidad de educación, pero esto no asegura un empleo en el área para el cual un estudiante se prepara.

En el mercado de trabajo hay dos opciones: trabajo por cuenta ajena y trabajo por cuenta propia. La búsqueda de empleo en ambos casos requiere una estrategia de planificación y ejecución de actividades que resulte idónea para su inserción laboral. (Sánchez, 2010).

El espíritu emprendedor lleva a muchos graduados en hotelería y turismo a poner en marcha su propio negocio en el sector. Normalmente los negocios en el sector privado son los que constituyen nuevas fuentes de empleo, reduciendo el índice de personas que no cuentan con un trabajo estable, incluyendo a desempleados con conocimiento en turismo que podría explotar su potencial en dichos negocios.

La inserción laboral en el Ecuador, a partir del período que comprende esta investigación tiene una expansión impresionante de la educación de tercer nivel, por lo que la oferta de personal altamente educado aumentó rápidamente y los graduados empezaron a sentir la presión de la competencia. (Abril, 2015). Según el autor, en el país se ha desarrollado positivamente la 
inserción laboral de los graduados debido a la buena educación recibida por las instituciones de tercer nivel, lo que ha ocasionado alta competencia entre dichos egresados, por lo que es importante la educación continua para poder diferenciarse de los demás.

Actualmente se está definiendo al mercado turístico como un buen sector para obtener un puesto de trabajo y poder desarrollarse profesionalmente, es decir, que los estudiantes y graduados en carreras de Turismo, si tienen posibilidades de involucrarse en el medio, sólo deben ser perseverantes en su búsqueda. "Los hoteles, restaurantes y la recreación ofrecen actualmente grandes posibilidades laborales y de crecimiento profesional a los jóvenes con disposición para aprender". (www.elempleo.com, 2012).

\section{Método de Investigación}

Se busca determinar el número de graduados hasta el 2015 en la carrera de Administración de Empresas Hoteleras de la Universidad Internacional del Ecuador que tuvieron y no tuvieron inconvenientes en su inserción laboral gracias a su formación académica, asi como determinar cuáles son las razones por las que el perfil del egresado ha influido positiva o negativamente en los graduados, ayudando o no en su inserción laboral.

Se realizaron encuestas on line aplicadas a los graduados en Administración de Empresas Hoteleras que ha tenido la Universidad Internacional del Ecuador desde que existe la extensión Guayaquil, al igual que la realización de entrevistas a profundidad hacia los gerentes generales, propietarios y jefes de Talento Humano de las principales cadenas hoteleras de mediana categoría en la ciudad de Guayaquil.

Resultados de las encuestas a los graduados del carrera de Administración de Empresas Hoteleras de Universidad Internacional del Ecuador, extensión Guayaquil.

Tabla $\mathrm{N}^{\circ}$ 5: Período de pasantías

\begin{tabular}{ccc}
\hline $\begin{array}{c}\text { Podría afirmar que para su } \\
\text { formación profesional el período de } \\
\text { prácticas o pasantías: }\end{array}$ & Cantidad & Porcentaje \\
\hline Ha aportado mucho & 9 & $39 \%$ \\
Ha aportado lo suficiente & 8 & $35 \%$ \\
Ha aportado muy poco & 5 & $22 \%$ \\
No ha aportado nada & 1 & $4 \%$ \\
Total & $\mathbf{2 3}$ & $\mathbf{1 0 0 \%}$ \\
\hline
\end{tabular}

En el gráfico se puede observar que el 39\% de los graduados indica que el período de pasantías laborales les ha aportado mucho en conocimientos y habilidades en su vida y carrera profesional, seguido por un 35\% que señalan que les ha aportado lo suficiente y tan sólo un $4 \%$ afirman que dichas prácticas no les han aportado en nada. Es muy importante que la Universidad y las demás instituciones tomen en cuenta dichas pasantías laborales para un mejor desarrollo del estudiante. 
Tabla $N^{\circ}$ 8: Empleo relacionado al sector turístico

\begin{tabular}{ccc}
\hline $\begin{array}{c}\text { El empleo que posee actualmente, ise encuentra } \\
\text { relacionado al sector de Turismo y Hotelería? }\end{array}$ & Cantidad & Porcentaje \\
\hline $\mathrm{Si}$ & 10 & $43 \%$ \\
No & 8 & $35 \%$ \\
No trabaja & 5 & $22 \%$ \\
Total & $\mathbf{2 3}$ & $\mathbf{1 0 0} \%$ \\
\hline
\end{tabular}

Según el gráfico se puede determinar que el $43 \%$ de los encuestados trabajan en áreas relacionadas al Turismo y Hotelería, un $35 \%$ se encuentran en otros campos y un $22 \%$ no trabajan. Es un resultado positivo el saber que la mayoría de los graduados han encontrado un puesto laboral dentro del campo para el cual realizaron su formación profesional.

Tabla $\mathbf{N}^{\circ}$ 9: Indique su lugar de trabajo

\begin{tabular}{|c|c|c|}
\hline Indique su lugar de trabajo: & Cantidad & Porcentaje \\
\hline LAN Ecuador & 3 & $13 \%$ \\
\hline Apart Hotel Kennedy & 1 & $4 \%$ \\
\hline Organización de eventos & 1 & $4 \%$ \\
\hline Comercio & 1 & $4 \%$ \\
\hline Expedia Lodging Partner Services & 1 & $4 \%$ \\
\hline Hotel Sonesta & 1 & $4 \%$ \\
\hline Club de Turismo, afiliación para viajeros & 1 & $4 \%$ \\
\hline Gerencia Avon & 1 & $4 \%$ \\
\hline Agencia de viajes Transvuelos & 1 & $4 \%$ \\
\hline Servicios & 1 & $4 \%$ \\
\hline Ventas & 1 & $4 \%$ \\
\hline Yanbal & 1 & $4 \%$ \\
\hline Comercio, venta de ropa & 1 & $4 \%$ \\
\hline Counter American Airlines & 1 & $4 \%$ \\
\hline Servicio al cliente & 1 & $4 \%$ \\
\hline Administrador Primax & 1 & $4 \%$ \\
\hline No trabaja & 5 & $22 \%$ \\
\hline Total & 23 & $100 \%$ \\
\hline
\end{tabular}

Según el gráfico se puede confirmar lo que se mencionó anteriormente, que la mayoría de los encuestados sí se encuentran trabajando en puestos relacionados al Turismo y Hotelería, como hoteles, aerolíneas y agencias de viajes, seguidos por aquellos que tienen su negocio propio, estén o no relacionados a la carrera. Algunos graduados que se encuentran trabajando en otras áreas que no son turísticas, prefieren mantener su puesto de trabajo estable, y por ello han desistido de buscar uno nuevo dentro de la Hotelería o Turismo. 


\section{Tabla $\mathrm{N}^{\circ}$ 11: Forma de ingreso al trabajo actual}

\begin{tabular}{|c|c|c|}
\hline Forma de ingreso al trabajo actual & Cantidad & Porcentaje \\
\hline Contactos personales/ familiar & 5 & $22 \%$ \\
\hline $\begin{array}{l}\text { Iniciativa personal (currículo, empresas de } \\
\text { selección) }\end{array}$ & 4 & $17 \%$ \\
\hline Anuncios (prensa, internet, etc.) & 4 & $17 \%$ \\
\hline Pasantías universitarias & 0 & $0 \%$ \\
\hline Bolsa de trabajo universitaria & 0 & $0 \%$ \\
\hline Negocio propio & 5 & $22 \%$ \\
\hline No trabaja & 5 & $22 \%$ \\
\hline Total & 23 & $100 \%$ \\
\hline
\end{tabular}

Según el gráfico se puede observar que el $22 \%$ de los encuestados ingresaron a su trabajo actual a través de contactos personales o familiares. En igual cantidad se encuentran los que poseen un negocio propio y aquellos que no trabajan. Un 17\% ingresaron a través de iniciativa personal con la entrega de un currículo, y en igual cantidad encontraron su puesto laboral gracias a anuncios publicados en prensa escrita, en páginas de internet o en redes sociales. Actualmente se conocen muchos casos en donde se ingresa al mundo laboral gracias a los contactos que se llegan a tener, lo que muchas veces resulta perjudicial para aquellos que intentan obtener un trabajo de la manera tradicional y sin ayuda de terceros.

\section{Tabla $\mathbf{N}^{\circ}$ 13: Tiempo para encontrar empleo}

\begin{tabular}{lcc}
\hline $\begin{array}{c}\text { ¿Cuánto tiempo le llevó encontrar } \\
\text { empleo desde la obtención de su título } \\
\text { universitario? }\end{array}$ & & \\
\hline Entre 1 a 60 Días & Cantidad & Porcentaje \\
Entre 2 a 12 Meses & 1 & $4 \%$ \\
Entre 1 Año en adelante & 3 & $13 \%$ \\
Aún no encuentra empleo & 2 & $9 \%$ \\
Ya se encontraba trabajando & 4 & $17 \%$ \\
\multicolumn{1}{r}{$\quad$ Total } & 13 & $57 \%$ \\
\hline
\end{tabular}

Según el gráfico se puede observar que el $57 \%$ de los encuestados ya se encontraban trabajando desde el momento que obtuvieron su título universitario, seguido por un $17 \%$ que aún no encuentra empleo, un 13\% que encontró un puesto laboral dentro de los 2 a 12 meses siguientes, un $9 \%$ que obtuvo uno luego de un año de graduado, y tan sólo un 4\% consiguió empleo dentro de los siguientes 60 días posteriores a la graduación. Nuevamente se observa que la mayoría ya tenía trabajo estable, frente al porcentaje de graduados que aún no encuentran empleo. El tiempo promedio de los que si obtuvieron trabajo se encuentra dentro del siguiente año posterior a la graduación. 
Tabla $N^{\circ}$ 15: Valoración de las competencias generales

\begin{tabular}{|c|c|c|c|c|c|c|c|c|}
\hline & \multicolumn{8}{|c|}{$\begin{array}{l}\text { Valore cada una de los siguientes competencios generales según el grado en el que la recibió en su formación académica, puntuando de } 1 \text { como "no la recibió" a } \\
\qquad 3 \text { como "la recibió en su totalidad", según su criterio. }\end{array}$} \\
\hline & & & & & & & & \\
\hline & COMPETENCIAS GENERALES & 1 & $\%$ & 2 & $\%$ & 3 & $\%$ & RESULIADO INDIVIDUAL \\
\hline & Capacidad de anâlisis y sintesis & 1 & $4 \%$ & 16 & $70 \%$ & 6 & $26 \%$ & LA RECIBIÓ PARCIALMENTE \\
\hline & Capacidad de organización y planificación & 1 & $4 \%$ & 15 & $65 \%$ & 7 & $30 \%$ & LA RECIBIÓ PARCIALMENTE \\
\hline & Comunicación oral y escrita & 1 & $4 \%$ & 13 & $57 \%$ & 9 & $39 \%$ & LA RECIBIÓ PARCIALMENTE \\
\hline & Conocimiento de lengua extranjera & 2 & $9 \%$ & 15 & $65 \%$ & 6 & $26 \%$ & LA RECIBIÓ PARCIALMENTE \\
\hline & Conocimiento de informática & 5 & $22 \%$ & 15 & $65 \%$ & 3 & $13 \%$ & LA RECIBIÓ PARCIALMENTE \\
\hline & Capacidad para resolución de problemas & 6 & $26 \%$ & 10 & $43 \%$ & 7 & $30 \%$ & LA RECIBIÓ PARCIALMENTE \\
\hline & Toma de decisiones & 4 & $17 \%$ & 12 & $52 \%$ & 7 & $30 \%$ & LA RECIBIÓ PARCIALMENTE \\
\hline & Capacidad para trabajar en equipo & 2 & $9 \%$ & 13 & $57 \%$ & 8 & $35 \%$ & LA RECIBIÓ PARCIALMENTE \\
\hline & Habilidades en las relaciones interpersonales & 2 & $9 \%$ & 15 & $65 \%$ & 6 & $26 \%$ & LA RECIBIÓ PARCIALMENTE \\
\hline & Razonamiento critico & 1 & $4 \%$ & 16 & $70 \%$ & 6 & $26 \%$ & LA RECIBIÓ PARCIALMENTE \\
\hline & Desarrollo de compromiso ético & 2 & $9 \%$ & 13 & $57 \%$ & 8 & $35 \%$ & LARECIBIÓ PARCIALMENTE \\
\hline & Aprendizaje autónomo & 3 & $13 \%$ & 16 & $70 \%$ & 4 & $17 \%$ & LA RECIBIÓ PARCIALMENTE \\
\hline & Adaptación a nuevas situaciones & 2 & $9 \%$ & 14 & $61 \%$ & 7 & $30 \%$ & LA RECIBIÓ PARCIALMENTE \\
\hline & Creatividad e innovación & 1 & $4 \%$ & 14 & $61 \%$ & 8 & $35 \%$ & LA RECIBIÓ PARCIALMENTE \\
\hline & Actitud de liderazgo & 3 & $13 \%$ & 11 & $48 \%$ & 9 & $39 \%$ & LA RECIBIÓ PARCIALMENTE \\
\hline & Iniciativa y espiritu emprendedor & 1 & $4 \%$ & 9 & $39 \%$ & 13 & $57 \%$ & LA RECIBIÓ EN SU TOTALIDAD \\
\hline & Motivación por la calidad & 0 & $0 \%$ & 13 & $57 \%$ & 10 & $43 \%$ & LA RECIBIÓ PARCIALMENTE \\
\hline & Conocimiento de recursos bibliográficos & 0 & $0 \%$ & 13 & $57 \%$ & 10 & $43 \%$ & LA RECIBIÓ PARCIALMENTE \\
\hline & Sensibilidad por temas medioambientales & 5 & $22 \%$ & 12 & $52 \%$ & 6 & $26 \%$ & LA RECIBIÓ PARCIALMENTE \\
\hline & Capacidad de aplicar los conocimientos en la práctica & 1 & $4 \%$ & 13 & $57 \%$ & 9 & $39 \%$ & LA RECIBIÓ PARCIALMENTE \\
\hline & Conocimientos básicos de la profesión & 3 & $13 \%$ & 6 & $26 \%$ & 14 & $61 \%$ & LA RECIBIÓ EN SU TOTALIDAD \\
\hline $\begin{array}{l}\text { TOTAL ENCUESTADOS: } 23 \\
\text { RESULTADO GLOBAL }\end{array}$ & $10 \%$ & & & $34 \%$ & & 10 & & \\
\hline
\end{tabular}

La tabulación de datos presentada, se puede conocer el grado en el que los estudiantes recibieron o adquirieron ciertas competencias que todo profesional debería poseer para poder desenvolverse en su campo. Los conocimientos básicos de la profesión ha sido la competencia más elevada y que ha sido recibida por los graduados en su totalidad en el transcurso de la carrera, seguido por iniciativa y el espíritu emprendedor que también la han percibido en su totalidad. El resto de competencias han sido recibidas parcialmente por parte de los encuestados, lo que no es ideal ya que indica que han sido enseñadas o transmitidas de manera incorrecta o que no se ha sabido llegar a los estudiantes mientras cursaban su carrera en la universidad, por ello es necesario reforzar dichas competencias para lograr un equilibrio total en los conocimientos y habilidades del graduado.

\section{Análisis e Interpretación de las entrevistas a profundidas hacia los empresarios Hoteleros}

Para el siguiente análisis, se realizaron entrevistas a siete empresarios hoteleros que administran o son propietarios de sus establecimientos de alojamiento. Los representantes de hoteles entrevistados y sus alojamientos pertenecen a la clasificación de Primera Categoría en la ciudad de Guayaquil y objeto de estudio.

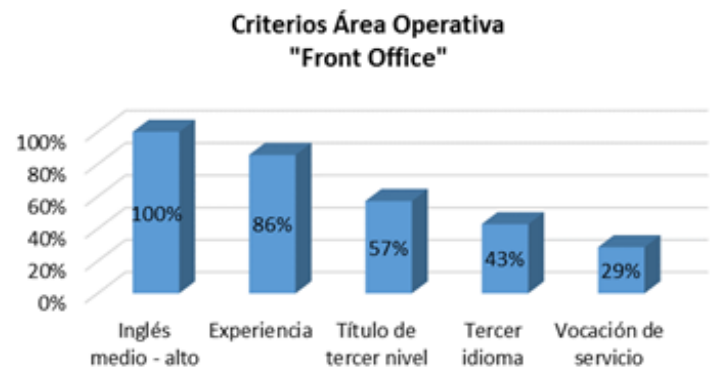

Gráfico $N^{\circ}$ 14: Criterios Área Operativa "Front Office" 
Para los empresarios hoteleros una de las principales competencias para vincularse al área de la recepción o Front office es el dominio del idioma inglés. Seguido de la experiencia con un $86 \%$, posteriormente y con un $57 \%$ de importancia es el título de tercer nivel.

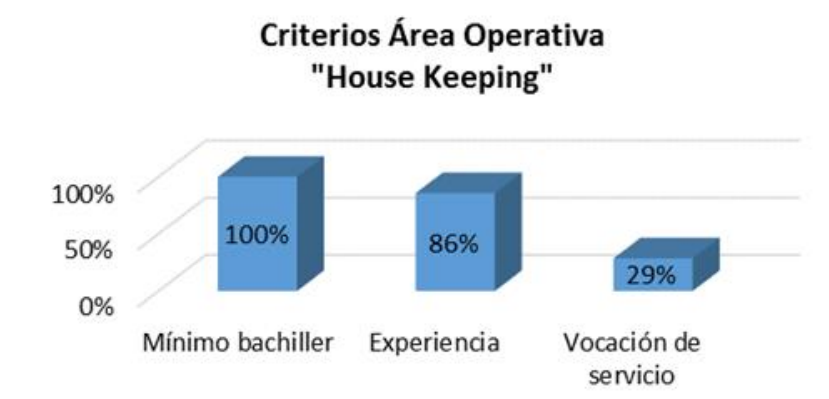

\section{Gráfico No 15: Criterios Área Operativa "House Keeping"}

En cuanto al área de Ama de llaves o House Keeping uno de los principales criterios para la contratación es que sean mínimo bachiller con un 100\%, seguido de experiencia del $86 \%$ y la vocación del servicio del $29 \%$.

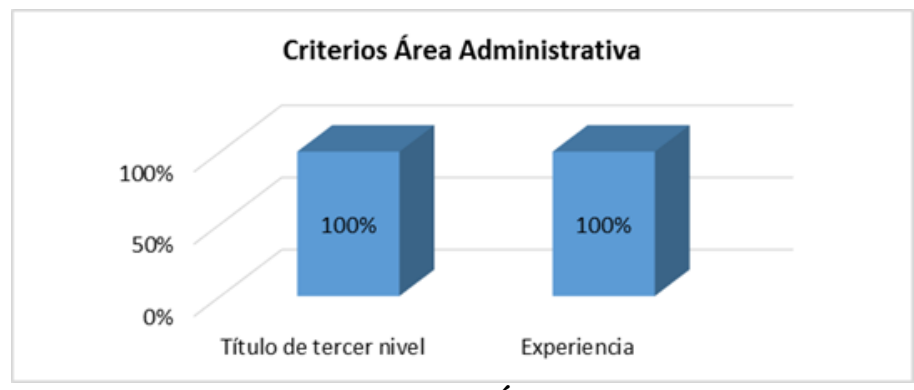

Gráfico $N^{\circ}$ 16: Criterios Área Administrativa

Entre los criterios de contratación para el área administrativa se requiere que posean título de tercer nivel e igual importancia es la experiencia.

\section{Vías para la búsqueda de aspirantes}

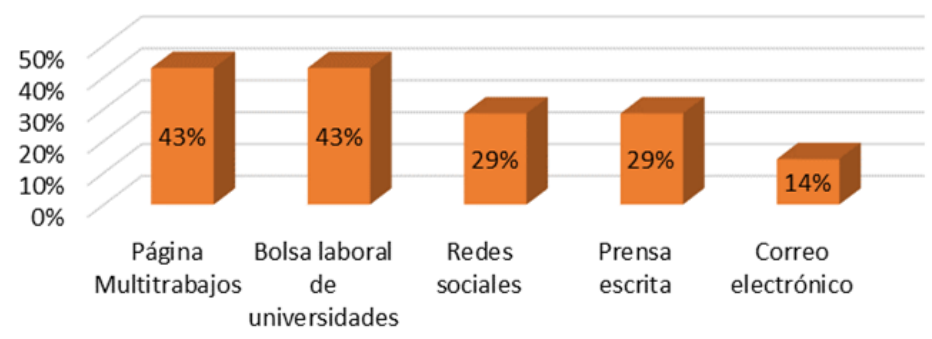

Gráfico $\mathbf{N}^{\circ}$ 17: Vías para la búsqueda de aspirantes

Para los representantes de hoteles las vías más comunes para la búsqueda de aspirantes son las páginas de multitrabajos y la bolsa laboral de las universidades con un $43 \%$ de 
porcentaje. Seguido del manejo de las redes sociales y prensa escrita con un $29 \%$ y un $14 \%$ el uso del correo electrónico.

\section{Contratación de graduados sin experiencia}

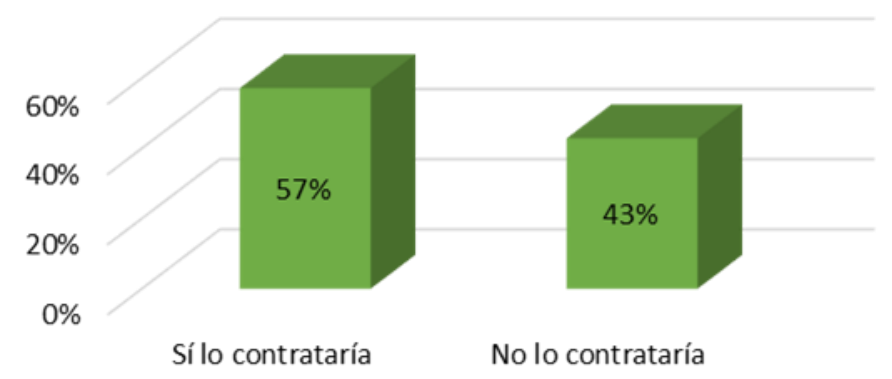

Gráfico $\mathrm{N}^{\circ}$ 19: Contratación de graduados sin experiencia

Para los empresarios Hoteleros entrevistados representan un $57 \%$ de importancia que los graduados posean experiencia para la contratación en sus establecimiento. Seguido con un $43 \%$ los empresarios hoteleros que no contratarían a graduados que no posean experiencia.

\section{Competencias que debe tener un colaborador hotelero}

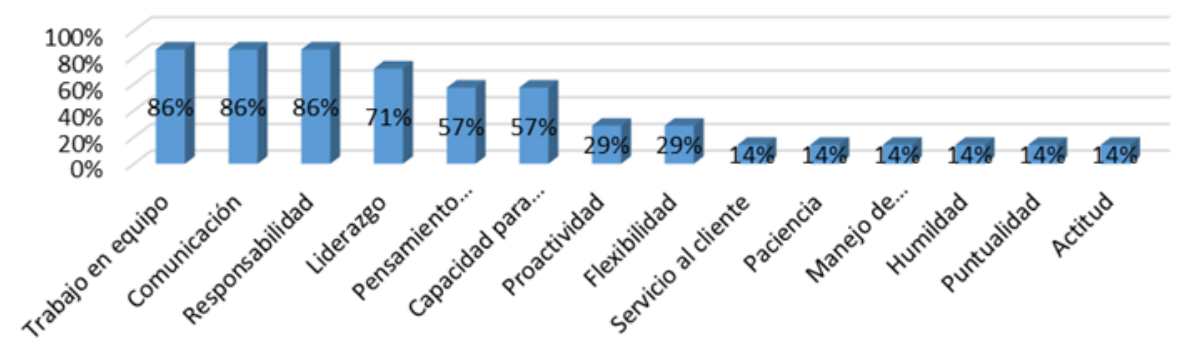

Gráfico $\mathrm{N}^{\circ}$ 22: Competencias que debe tener un colaborador hotelero

Para los empresarios hoteleros es importante que el trabajo en equipo sea una de las principales competencia que debe poseer un profesional hotelero, seguido de la comunicación, responsabilidad con un $86 \%$ de importancia. En porcentajes inferiores el liderazgo, pensamiento creativo, entre otros.

\section{Contratación de pasantes}

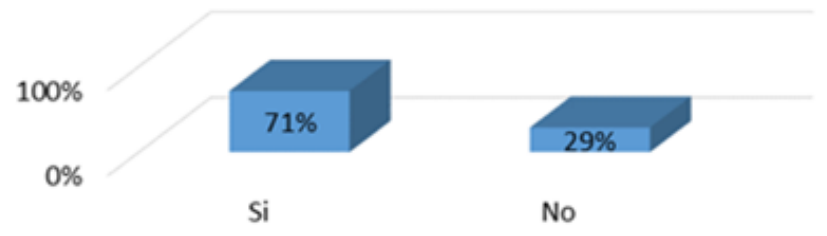

Gráfico $\mathbf{N}^{\circ}$ 26: Contratación de pasantes 


\section{¿Existen pasantes que se hayan quedado trabajando para el hotel?}

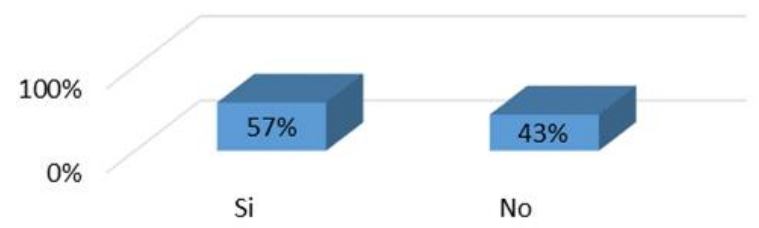

Gráfico $N^{\circ}$ 27: ¿Existen pasantes que se hayan quedado trabajando para el hotel?

El 57\% de los empresarios hoteleros han contratado los pasantes que han recibido en su hotel. Mientras que el $43 \%$ de los empresarios no cuentan con pasantes que se hayan quedado en su establecimiento.

\section{Carencia formativa en universidades de Hotelería}

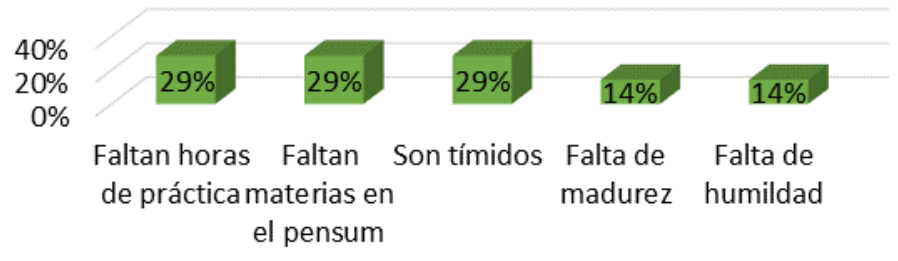

Gráfico $\mathrm{N}^{\circ}$ 28: Carencia formativa en universidades de Hotelería

Discusión. El perfil profesional de un graduado en Administración de Empresas Hoteleras sí influye en gran parte al momento de ser contratado por una determinada empresa turística u hotelera, debido a que, el graduado debe contar con conocimientos y habilidades (competencias) básicas y específicas para desempeñar un puesto de trabajo relacionado a la carrera, como por ejemplo conceptos básicos y terminología profesional, capacidad para resolver problemas, responsabilidad, comunicación, liderazgo, flexibilidad, servicio al cliente y trabajo en equipo, las cuales son valoradas positivamente por los empleadores en el sector de la Hotelería y Turismo y como hemos podido apreciar en los cuadros.

El período de pasantías obtenido a nivel general, les ha aportado gran valor a su vida laboral, seguido en igual porcentaje a quienes les ha aportado lo suficiente, no llegando al nivel óptimo esperado. Y también las competencias generales que no han sido totalmente percibidas por los graduados mientras fueron estudiantes, rescatándose un nivel óptimo de conocimientos básicos de la profesión. Aun así, la mayoría de graduados si posee un puesto laboral fijo, pero no todos ellos dentro del sector turístico u hotelero.

Dichos resultados obtenidos aportarán un conocimiento actualizado, sobre las falencias detectadas en los perfiles de los graduados en Administración de Empresas Hoteleras, que aportarán a rediseños en el perfil por competencias de la carrera que esté más ligado a la misma y a lo que se encuentran solicitando actualmente los empleadores del sector hotelero.

\section{Conclusiones}


El perfil profesional de un graduado en Administración de Empresas Hoteleras de la Universidad Internacional del Ecuador, sede Guayaquil influye en la contratación laboral de los mismos, debido a que, aunque la mayoría de ellos poseen un trabajo estable, no todos están relacionados al sector turístico, existiendo una falta de coherencia entre lo estudiado y el área de desarrollo profesional.

Las encuestas realizadas a los estudiantes indican que el $43 \%$ de los graduados en Administración de Empresas Hoteleras de la Universidad Internacional del Ecuador extensión Guayaquil, se encuentran trabajando en el área turística y hotelera. Coincidiendo los estudiantes en la importancia que les significan las materias de la malla curricular de la carrera y el grado en el que han recibido las competencias generales en su vida universitaria como las practicas pre profesionales.

Las entrevistas aplicadas a los gerentes generales, propietarios y jefes de talento humano de las principales cadenas hoteleras de mediana categoría en la ciudad de Guayaquil, determinan la inserción laboral de un graduado en la carrera de Administración de Empresas Hoteleras son las competencias generales adquiridas en la universidad, el período de pasantías o prácticas profesionales, que equivale a experiencia laboral, y los conocimientos recibidos (teóricos y prácticos) en las materias de la malla curricular de la carrera. Los resultados de la investigación coadyuvan a un futuro rediseño en el perfil por competencias que la carrera de Administración de Empresas Hoteleras crea necesaria realizar, tomando en cuenta los factores antes mencionados.

\section{Bibliografía}

Abril, J. (2015). Proceso de formación e inserción laboral de los profesionales en Turismo de la Universidad del Azuay de la Cohorte 2001 y su influencia en la economía del Azuay. Cuenca, Azuay, Ecuador. Obtenido de http://dspace.uazuay.edu.ec/bitstream/datos/4985/1/11423.pdf

Acuña, K., \& Mozombite, C. (6 de Diciembre de 2013). Propuesta del perfil del recién egresado de Administración de Empresas basado en sus competencias genéricas y específicas, que requieren hoy en día las principales empresas del departamento de Lambayeque. Chiclayo, Perú. Obtenido de http://tesis.usat.edu.pe/jspui/bitstream/123456789/277/1/TL_MozombiteGrandezCarlos_ Acu\%C3\%B1aHernandezKattia.pdf

Albornoz, C., \& Escudero, F. (2007). Competencias profesionales en la formación del Ingeniero Civil Acústico. Valdivia, Chile. Obtenido de http://cybertesis.uach.cl/tesis/uach/2007/bmfcia339c/doc/bmfcia339c.pdf

Antofagasta, U. d. (s.f.). Cuestionario para egresados. Chile. Obtenido de https://www.uantof.cl/acreding/Cuestionarios/Cuestionario\%20para\%20egresados.pdf

Araujo, D. F. (Abril de 2009). La enseñanza superior e inserción laboral de los profesionales con formación en turismo y áreas afines: El caso del Polo Turístico Salvador e Entorno". Las 
Palmas de Gran Canaria. Obtenido de http://acceda.ulpgc.es/bitstream/10553/3465/1/Tesis_Domira_Fernandes_Araujo.pdf

Ávila, U. C. (2009). Encuesta para egresados Ingeniero Agrícola. Obtenido de https://www.ucavila.es/DocumentosAneca/AnexosGradoIngAgropecuaria.pdf

Ayala, M. d., Castillo, I., \& Hernández, C. (Agosto de 2011). Perfil del profesional en Administración de Empresas en el área de Recursos Humanos de la Universidad de El Salvador. San Salvador, El Salvador. Obtenido de http://ri.ues.edu.sv/1103/1/Perfil_Profesional_En_Administracion_De_Empresas_En_El_ Area_De_Recursos_Humanos.Pdf

Baigorria, E. (2012). El proceso de inserción laboral de los inmigrantes en el sector turístico. Neuquén, Argentina. Obtenido de http://170.210.83.98:8080/jspui/bitstream/123456789/323/1/baigorria_proceso_insercion _laboral_inmigrantes_sector_turismo.pdf

Beier, G. (2014). Inserción laboral de los egresados de la Licenciatura en Comercio Internacional de la UNLu. Obtenido de http://www.frd.utn.edu.ar/sites/default/files/CarrerasPos/Tesis\%20Docencia\%20$\%$ 20BEIER.pdf

Carrión, L. (2013). Diseño y elaboración de una herramienta técnico-operativa para la guía turística en la ciudad de Loja. Loja, Loja, Ecuador. Obtenido de http://repositorio.uide.edu.ec/bitstream/37000/599/1/T-UIDE-0549.pdf

Castro, F., \& Alabart, Y. (2011). Propuesta del Perfil de Cargos por Competencias para un Gerente General, aplicable a Hoteles de segunda categoría en la Ciudad de Guayaquil. Guayaquil, Ecuador. Obtenido de http://www.uteg.edu.ec/_documentos/file/pdf/perfil-de-cargos-porcompetencia.pdf

Crous-Costa, N., \& Vidal-Casellas, D. (2013). La experiencia laboral durante los estudios universitarios: El rol las prácticas en los estudios de turismo. Obtenido de http://dugidoc.udg.edu/bitstream/handle/10256/9121/ExperienciaLaboral.pdf?sequence $=1$

diarioelpueblo.com. (13 de Septiembre de 2015). La inserción laboral de los jóvenes. Obtenido de http://www.diarioelpueblo.com.uy/informe/la-insercion-laboral-de-losjovenes.html

Dieckow, L. M., \& Kremar, A. A. (25 de Marzo de 2014). El mercado de trabajo y empleo de profesionales en turismo en Misiones. Análisis de la inserción, movilidad y nivel de salarios. Obtenido de http://argos.fhycs.unam.edu.ar/bitstream/handle/123456789/157/321\%20avance\%2011\% 20DIECKOW\%20Mercado\%20de\%20Trabajo\%20del\%20Turismo\%20en\%20Mnes.pdf? sequence $=1 \&$ isAllowed $=\mathrm{y}$ 
Fonseca, M. (2005). Políticas para o encino superior en Turismo: un estudio sobre un curso de graduaos en Horizonte. Obtenido de http://www.biblioteca.pucminas.br/teses/Educacao_FonsecaMM_1r.pdf

García, C. (31 de Agosto de 2009). Comportamiento del mercado laboral en México. Santiago de Chile, Chile. Obtenido de http://repositorio.uchile.cl/tesis/uchile/2009/csgarcia_c/pdfAmont/cs-garcia_c.pdf?origin=publication_detail

García, M. R. (27 de Diciembre de 2006). Las competencias de los alumnos universitarios. Zaragoza, España. Obtenido de http://www.redalyc.org/pdf/274/27411311013.pdf

Gómez, V. (Junio de 2009). Desempeño laboral del licenciado en Turismo de la Universidad de Oriente, según la opinión del sector empresarial turístico privado de la Isla de Margarita, Estado Nueva Esparta. Isla Margarita, Venezuela. Obtenido de http://mingaonline.uach.cl/scielo.php?pid=S0718-

$64282009000200003 \&$ script $=$ sci_arttext

images.slideplayer.es. (s.f.). La personalidad del estudiante. Obtenido de http://images.slideplayer.es/7/1804165/slides/slide_4.jpg

images.slideplayer.es. (s.f.). Variables y dimensiones que influyen en el proceso de inserción. Obtenido de http://images.slideplayer.es/1/39582/slides/slide_5.jpg

Jara, A. (Agosto de 2010). Definición de perfiles del recurso humano para la administración de proyectos en la subgerencia de tecnologías de información y comunicaciones de la CCSS. San José, Costa Rica. Obtenido de http://www.uci.ac.cr/Biblioteca/Tesis/Pfgmap854.pdf Llanos, J. R. (2008). Relación del perfil profesional y el plan de estudios con el desempeño docente; de los egresados de la especialidad de Biología y Química de la Universidad Nacional Hermilio Valdizán de Huánuco. Lima, Perú. Obtenido de http://cybertesis.unmsm.edu.pe/bitstream/cybertesis/2414/1/Llanos_mj.pdf

Malán, M. (Noviembre de 2011). Inserción laboral de personas con síndrome de Down en hoteles de cuatro y cinco estrellas del distrito metropolitano de Quito. Quito, Pichincha, Ecuador. Obtenido de http://repositorio.uisrael.edu.ec/bitstream/47000/241/1/UISRAEL-ECADMH-378.242-536.pdf

Martínez, R. (2005). La inserción laboral de los universitarios a través de las prácticas en empresas. Granada, España. Obtenido de dialnet.unirioja.es/descarga/articulo/743415.pdf

Ministerio de Turismo. (27 de Noviembre de 2014). Ecuador se fortalece con inversiones en el sector turístico. Obtenido de http://www.turismo.gob.ec/ecuador-se-fortalece-coninversiones-en-el-sector-turistico/

Ministerio de Turismo. (Mayo de 2015). Proyecto para la consolidación, regulación y control turístico. Obtenido de http://www.turismo.gob.ec/wpcontent/uploads/2015/05/Documento-Proyecto-Consolidacion-Regulaci\%C3\%B3n-yControl-T.-dic-2014.pdf 
Nayarit, U. A. (Enero de 2014). Programa académico de la Licenciatura en Turismo. Tepic, Nayarit, México. Obtenido de http://www.uat.uan.edu.mx/archivos/2014/ene/Proyecto_Curricular_Turismo_2013.pdf

Ortiz, G. M. (2013). Perfiles por competencias para los guías turísticos del Ecuador. Quito, Pichincha, Ecuador. Obtenido de http://repositorio.flacsoandes.edu.ec/bitstream/10469/5915/2/Tflacso-2013gmog.pdf

Pavié, A. (2012). Las competencias del profesorado de lengua castellana y comunicaciones en Chile: Aportaciones a la formación inicial. Obtenido de http://uvadoc.uva.es/bitstream/10324/2794/1/Tesis297-130508.pdf

Peñaloza, L., Medina, J. L., Herrera, A., \& Vargas, R. (12 de Abril de 2011). La profesión turística: ejes de explicación en su relación con la formación académica. Obtenido de http://www.scielo.org.mx/scielo.php?pid=S0185-27602011000400005\&script=sci_arttext

Pereira, J. (2012). La formación profesional y el mercado laboral de los técnicos agrícolas del Instituto Federal de Educación, Ciencia y Tecnología del estado de Maranhao Campus Sao Luis Maracaná. Maranhao - Brasil. Madrid, España. Obtenido de http://dspace.uah.es/dspace/bitstream/handle/10017/15301/Tesis\%20doctoral.\%20Jandira $\% 20$ Pereira\%20SouzaDIC\%20(1).pdf?sequence $=1$

Sánchez, M. (2010). Factores determinantes para la inserción y distintas formas de participación en el mercado laboral gallego. A Coruña, España. Obtenido de http://ruc.udc.es/bitstream/2183/9876/2/SanchezSellero_MariaCarmen_TD_2010.pdf

scielo.org.co. (s.f.). Dimensiones inserción laboral. Obtenido de http://www.scielo.org.co/img/revistas/rfce/v21n1/v21n1a05-1.jpg

Segundo, M. (Enero de 2009). Inserción al mercado laboral de los profesionistas de la UACJ: Desde el enfoque de la política de ampliación de cobertura. México D.F., México. Obtenido de http://conocimientoabierto.flacso.edu.mx/medios/tesis/segundo_me.pdf

Souza, T. R., \& Luce, C. (2007). Inserción profesional de los licenciados en turismo: El caso de la Universidad Federal de Minas Gerais (Brasil) entre 2005 y 2007. Obtenido de http://www.scielo.org.ar/scielo.php?script=sci_arttext\&pid=S1851-17322010000600013 www.elempleo.com. (2 de Marzo de 2012). Sector hotelero y turístico: gran generador de empleo. Obtenido de http://www.elempleo.com/colombia/consejos_profesionales/sectorhotelero-y-turnustico-gran-generador-de-empleo-en-el-panus-------/11176283

www.med.unne.edu.ar. (s.f.). Calculadora para obtener el tamaño de una muestra. Obtenido de http://www.med.unne.edu.ar/biblioteca/calculos/calculadora.htm

Zabalza, M. Á. (2007). Competencias docentes del profesorado Universitario: calidad y desarrollo profesional. Madrid: Narcea, S.A. de Ediciones. 
Zalba, E. (2004). Seguimiento de la inserción laboral y el desempeño profesional de los graduados de la Uncuyo. Mendoza, Argentina. Obtenido de http://bdigital.uncu.edu.ar/objetos_digitales/798/ZalbaFinalGral2003-2004.pdf 